Communications in Physics, Vol. 24, No. 3S2 (2014), pp. 89-100

DOI:10.15625/0868-3166/24/3S2/5054

\title{
POSSIBILITY OF GENERATION AND AMPLIFICATION TO HIGH POWER OF ULTRAVIOLET CE:LICAF LASER SHORT PULSES
}

\author{
PHAM HONG MINH, PHAM VAN DUONG, PHAM HUY THONG, DO QUOC KHANH, \\ AND NGUYEN DAI HUNG \\ Institute of Physics, Vietnam Academy of Science and Technology \\ NOBUHIKO SARUKURA \\ Institute of Laser Engineering, Osaka University, Suita Osaka 565-0871, Japan \\ E-mail:phminh@iop.vast.ac.vn
}

Received 20 June 2014

Accepted for publication 20 August 2014

\begin{abstract}
We review the recent progresses in generation and amplification of ultraviolet laser emissions using $\mathrm{Ce}^{3+}: \mathrm{LiCaAlF}_{6}$ (Ce:LiCAF) material as a gain medium. Basing on comparative studies, we have investigated improvements and proposed possibilities to generate and amplify ultraviolet short-pulse Ce:LiCAF laser emission to high peak power of terawatt.
\end{abstract}

Keywords: Ce:LiCAF crystal, ultraviolet, short pulse, laser generation, amplification.

\section{INTRODUCTION}

High power laser light sources operating in the ultraviolet (UV) spectral region have received a great deal of interest for numerous applications in science and technology such as semiconductor processing, micromachining, remote sensing with Lidar, engine combustion diagnostics, spectroscopy, medicinal and biological applications... [1-6]. Hence, there is a great deal of interest for the development of high-power, all-solid-state UV lasers of convenient operating procedures. The existing means to produce tunable UV laser emission is mainly based on frequency conversion using nonlinear crystals such as doubling, tripling and/or mixing of tunable laser emission generated from the primary tunable lasers. However, this way has several disadvantages such as its complexity, high cost, limited spectral bandwidth and tunability, inefficiency, inconvenience and unreliability for airborne measurement in flight or aboard spacecraft applications [1].

The use of rare earth-doped fluorides as active media has been shown to be an excellent way to produce UV laser emission directly. There have been emerged the ${ }^{1}$ convenient and compact laser sources based on the dipole-allowed inter - configurational transitions of $\mathrm{Ce}^{3+}$ ions doped in wide band-gap fluorides [7-10]. For example, Trivalent Cerium $\left(\mathrm{Ce}^{3+}\right)$ doped fluorides such

${ }^{1}$ change ... "This has emerged" to "There have been emerged the" 
as $\mathrm{LiCaAlF}_{6}(\mathrm{LiCAF})$ and $\mathrm{LiSrAlF}_{6}(\mathrm{LiSAF})$ were researched and developed for the direct and efficient generation of tunable UV laser emission [9-11]. Among these crystals, Ce:LiCAF is an especially attractive solid-state laser medium because of its transparency, tolerance to laserinduced damage owing to the absence of solarization effects, and ability to be directly pumped at $266 \mathrm{~nm}$ by the fourth harmonic generation of Nd:YAG laser [7-14]. In addition to broad UV tunability (from 280 to $325 \mathrm{~nm}$ ), the cerium lasers have considerable potential for ultrafast pulse generation and amplification [10-15]. For example, the gain spectrum of Ce:LiCAF is broad enough to produce laser pulses as short as 3 -fs, its sufficiently large effective gain cross section $\left(6.0 \times 10^{-18} \mathrm{~cm}^{2}\right)$ and its high saturation fluence $\left(115 \mathrm{~mJ} / \mathrm{cm}^{2}\right)$ are favorable for laser oscillators and power amplifiers [10]. Despite the remarkable performance of Ce:LiCAF crystal, however, Ce:LiCAF laser - amplifier has not yet produced UV pulsed laser emission of terawatt (TW) peak power level. Therefore, we have been continuously geared towards the research and development of short UV laser pulse generation and amplification as well as UV laser media.

This paper presents the recent progresses in generation and amplifications of UV laser emissions using Ce:LiCAF crystal as a gain medium. Additionally, we have introduced our activities in the research and development of UV short pulse generation and amplification, and proposed a Ce:LiCAF laser - amplifier system of producing UV laser pulses of terawatt peak power.

\section{PROPERTIES OF A Ce:LiCAF CRYSTAL ACTIVE MEDIUM}
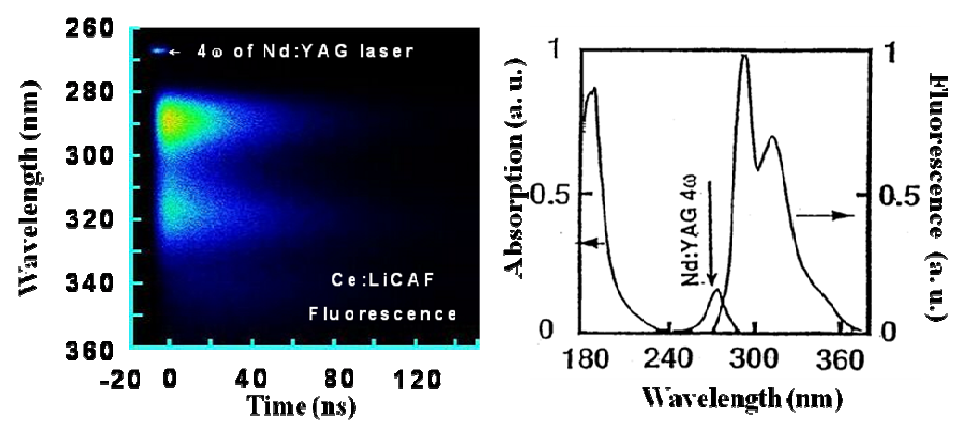

Fig. 1. (a) Streak camera image of the fluorescence of Ce:LiCAF excited at $266 \mathrm{~nm}$ by the $4^{\text {th }}$ harmonic of a Nd:YAG laser, (b) absorption and fluorescence spectra of a Ce:LiCAF crystal $[1,12]$.

The first reports on ${ }^{2}$ Ce:LiCAF crystal as an active medium in UV wavelength range was given by Dubinskii, et al. in 1993, which could be optically excited at $266 \mathrm{~nm}$ by the fourth harmonic generation of a Nd:YAG laser [7, 8]. Remarkably, no solarization effect was observed in this crystal. The Ce:LiCAF fluorescence and absorption spectra are shown in Fig. 1, presenting the nearly two-hump shaped characteristic of $\mathrm{Ce}^{3+}$ ions in most known hosts due to the allowed $5 d-4 f$ transitions terminating at the ${ }^{2} \mathrm{~F}_{5 / 2}$ and ${ }^{2} \mathrm{~F}_{7 / 2}$ components of the spin-orbit split ground levels [9]. The properties of Ce:LiCAF crystal are summarized in Table 1.

\footnotetext{
2 change ... "for" to "on"
} 
Table 1. Material properties of Ce:LiCAF crystal $[7,9,10,16]$.

\begin{tabular}{|l|l|}
\hline $\mathrm{Ce}^{3+}$ doping concentration & $1 \mathrm{~mol} \%[16]$ \\
\hline Doping density, $N$ & $5 \times 10^{17} \mathrm{~cm}^{-3}[16]$ \\
\hline $\begin{array}{l}\text { Absorption coefficient at } 266 \mathrm{~nm} \text { (wave- } \\
\text { length of pump laser) }\end{array}$ & $4 \mathrm{~cm}^{-1}[16]$ \\
\hline Absorption cross section at $266 \mathrm{~nm}$ & $7.3 \times 10^{-18} \mathrm{~cm}^{-2}[9,16]$ \\
\hline Absorption cross-section, $\sigma_{a i}$ & $2.606 \times 10^{-19} \mathrm{~cm}^{-2}$ at $290 \mathrm{~nm}[9,16]$ \\
\hline Emission cross-section, $\sigma_{e i}$ & $9.6 \times 10^{-18} \mathrm{~cm}^{-2}$ at $290 \mathrm{~nm}[9,16]$ \\
\hline ESA cross section at $266 \mathrm{~nm}$ & $5.5 \times 10^{-18} \mathrm{~cm}^{-2}[9]$ \\
\hline ESA cross section at $290 \mathrm{~nm}$ & $3.6 \times 10^{-18} \mathrm{~cm}^{-2}[9]$ \\
\hline Gain cross section & $6 \times 10^{-18} \mathrm{~cm}^{-2}[9]$ \\
\hline Refractive index, $n$ & $1.41[7,9,13]$ \\
\hline Fluorescence lifetime, $\tau$ & $25 \pm 2 \mathrm{~ns}[9]$ \\
\hline Emission spectrum & $275 \mathrm{~nm} \mathrm{to} 330 \mathrm{~nm}[9]$ \\
\hline Gain spectrum & 280 to $320 \mathrm{~nm}[7,10,16]$ \\
\hline Saturation fluence & $115 \mathrm{~mJ} / \mathrm{cm}^{2}[7,9,10]$ \\
\hline
\end{tabular}

Basing on the material properties and parameters presented in Table 1, some comments on Ce:LiCAF as an active medium can be given as follows:

Ce:LiCAF has a strong absorption at $266 \mathrm{~nm}$, hence, the fourth harmonic generation of $\mathrm{Nd}$ :YAG laser is an ideal pump source.

Ce:LiCAF has a gain broadband from $280 \mathrm{~nm}$ to $320 \mathrm{~nm}$. This is attractive for ultraviolet short pulse generation and applications.

Ce:LiCAF has an effective gain cross-section $\left(6 \times 10^{-18} \mathrm{~cm}^{2}\right)$ sufficiently higher that of Ti:sapphire.

Ce:LiCAF has higher saturation fluence $\left(115 \mathrm{~mJ} / \mathrm{cm}^{2}\right)$ than organic dyes. The thermal conductivity of Ce:LiCAF are low and anisotropic in nature $\left(3.09-2.9 \mathrm{~W} / \mathrm{m}^{0} \mathrm{C}\right)$. This makes it attractive for designing power UV laser amplifiers.

The fluorescence lifetime of Ce:LiCAF was reported to be $25 \mathrm{~ns}$. It would be too short for constructing regenerative amplifier; it is long enough for designing multi-pass amplifiers and also long enough for electronic delay control of the amplifier timing.

\section{Ce:LiCAF CRYSTALS AS PROSPECTIVE ULTRAVIOLET ACTIVE MEDIA}

After the first reports of direct generation of UV laser light from Ce:LiCAF, broadly tunable laser operations of Ce:LiCAF lasers were investigated by other groups $[10,11,18,19]$. Singleprism tunability of transversely and longitudinally pumped Ce:LiCAF lasers was achieved. For example, tunability of laser wavelength was achieved with an intra-cavity prisms or Littrow-prisms, as shown in Fig. 2 [18]. The broadest tuning range of Ce:LiCAF lasers was accomplished from $280 \mathrm{~nm}$ to $316 \mathrm{~nm}[18]$.

Ce:LiCAF crystal has considerable potential for short pulse generation. Several different methods to produce short pulses from Ce:LiCAF lasers have been proposed [10, 14, 15, 18-20]. 


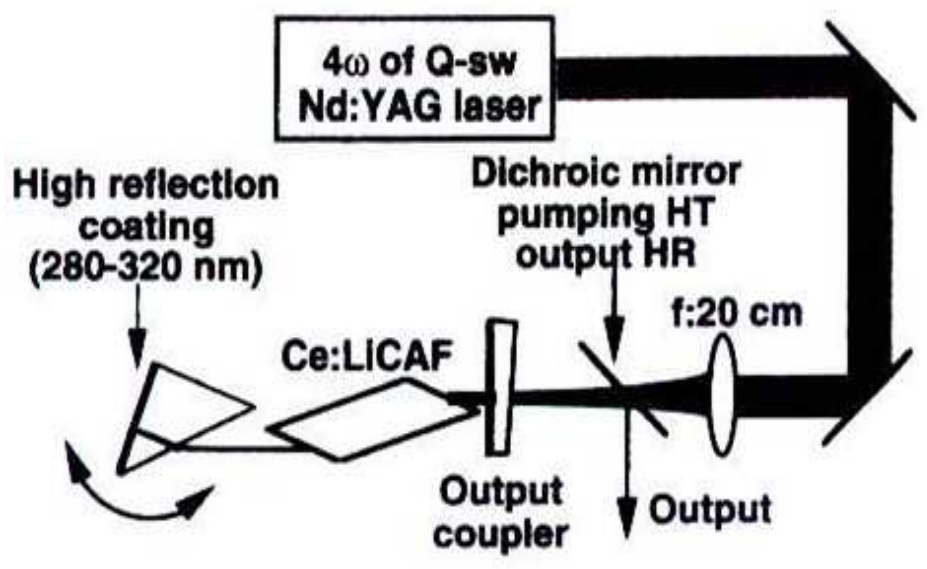

Fig. 2. Experimental setup of the tunable Ce:LiCAF laser oscillator pumped by the fourth hamonics generation of a Q-switched Nd:YAG laser [18].

Using the method of self - injection - seeded pulse, generation of a sub-nanosecond UV pulse train from a Ce:LiCAF laser was firstly reported by author's group [14, 15]. In the experiment, a laser master oscillator and a feedback laser cavity of $1.1 \mathrm{~m}$ long were used [14]. The laser master oscillator was formed by a flat high reflection mirror and a flat output coupler of $80 \%$ transmission. A $1 \%$ doped, $5 \mathrm{~mm}$ cubic Ce:LiCAF crystal was used as an active medium without any dielectrics coatings on the polished surfaces. In a combined operation with the feedback laser cavity, UV laser pulses of 600 ps were generated from the Ce:LiCAF laser master oscillator and transformed to a sub-nanosecond pulse train.

The generation of short UV pulse from Ce:LiCAF lasers has been interested in the cavitytransient method. This method is quite simple and could provide a pulse-compression factor as high as 10 with respect to the duration of pump pulse. Using suitably selected parameters of the resonator and pump energy, short laser pulse generation were demonstrated to be available in different lasers. For example, sub-nanosecond pulse generation without sacrify tunability was obtained in an all solid state Ce:LiCAF laser pumped by the fourth harmonic generation of a nanosecond Q-switched Nd:YAG laser [18]. The laser was modified by using a Brewster-cut 10 $\mathrm{mm}$ Ce:LiCAF crystal and a half-cut Brewster prism, which was high reflection-coated at one face and used as an end mirror. To obtain single and shorter laser pulse of high energy, a low-Q and short resonator of this Ce:LiCAF laser was developed by using an output coupler of a high transmission of $75 \%$. The pumping nanosecond pulse energy could be varied from 2 to $4 \mathrm{~mJ}$ to enable the generation of single UV short pulses with pulse energy of about $170 \mu \mathrm{J}$. The shortest pulse duration obtained with this laser configuration is $700 \mathrm{ps}$. The wavelength tuning range was experimentally demonstrated from $281 \mathrm{~nm}$ to $297 \mathrm{~nm}$ [18].

Recently, we numerically investigate the characteristics of UV laser emission from a Ce:LiCAF laser using a rate equation system extended to multi-wavelengths. We demonstrated that picosecond UV laser emission is obtainable from the Ce:LiCAF laser using appropriate pumping and 
resonator parameters (Q-factor). Our results have successfully reproduced experimental observations and showed the dependence of the laser pulse duration on the pumping energy and cavity parameters, hence determining the optimum cavity design of a simple, short-pulse Ce:LiCAF UV laser. Particularly, we simulated the experimental results by using the same parameters as in the works done by Liu et al [18]. The Ce:LiCAF crystal length, $l=10 \mathrm{~mm} ; N=5 \times 10^{17} \mathrm{~cm}^{-3}$ for $1 \mathrm{~mol} \%$ doping concentration; index of refraction, $n=1.41$; cavity length, $L=25 \mathrm{~mm}$; end mirror reflectivity, $R_{1}=100 \%$; output coupler reflectivity, $R_{2}=30 \%$; pump beam radius, $\mathrm{r}=1 \mathrm{~mm}$, pumping energy, $E_{\text {pump }}=3.6 \mathrm{~mJ} \mathrm{~mm}$ and 5 nanosecond of laser pump from the fourth harmonics $(266 \mathrm{~nm})$ of an Nd:YAG laser operating at $10 \mathrm{~Hz}$ repetition. The spectro-temporal evolution of this low-Q, short cavity Ce:LiCAF laser pulse emission is obtained and presented in Fig. 3. This shows a maximum gain coefficient is achieved at $288.5 \mathrm{~nm}$ and a maximum laser intensity can be observed at around $288.5 \mathrm{~nm}$. This characteristic of Ce:LiCAF laser emission is consistent with the experimental observation, regardless of doping concentration. The computed pulse duration is around $701 \mathrm{ps}$. The investigations will be presented in detail elsewhere.
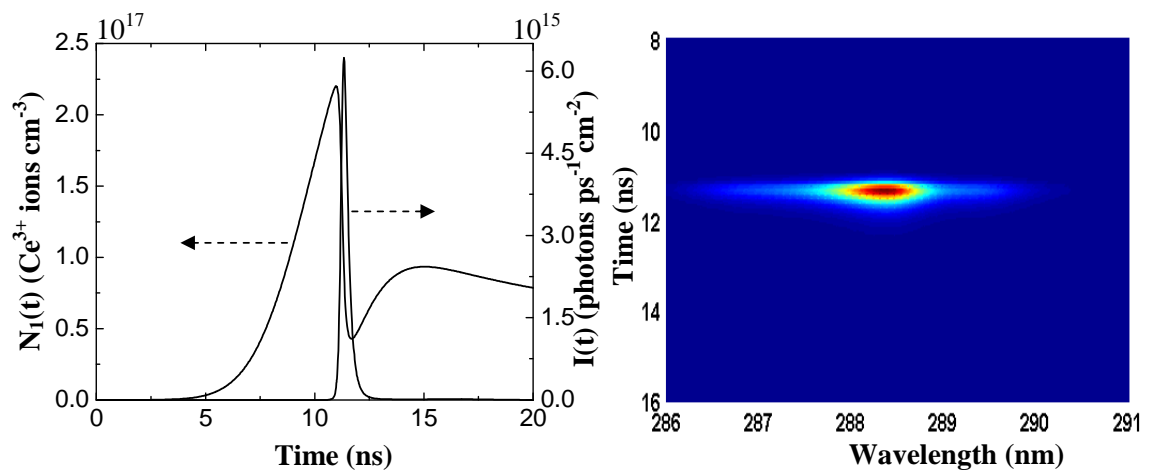

Fig. 3. a) Generation of a short laser pulse of $349 \mathrm{ps}$, b) spectro-temporal evolution of the broadband Ce:LiCAF laser pulse from the low- $\mathrm{Q}\left(\mathrm{R}_{2}=30 \%\right)$, short cavity $(\mathrm{L}=25 \mathrm{~mm})$ oscillator. The 10 -mm-long, $1 \mathrm{~mol} \% \mathrm{Ce}^{3+}$-doped LiCAF crystal pumped with a $266 \mathrm{~nm}$, $5 \mathrm{~ns}$.

The rate equations clearly show that the rate of change of excited state population density and laser intensity strongly depend on the pump energy. Therefore, we solved the rate equations for varying pump energies from 3.6 to $8 \mathrm{~mJ}$ in order to determine its effect on the pulse duration of the Ce:LiCAF laser emission. These parameters were the same as the experimental parameters [18], i.e. crystal length, $l=10 \mathrm{~mm} ; \mathrm{N}=5 \times 10^{17} \mathrm{~cm}^{-3}$ for $1 \mathrm{~mol} \%$ doping concentration; cavity length, $\mathrm{L}=25 \mathrm{~mm}$; end mirror reflectivity, $\mathrm{R}_{1}=100 \%$; output coupler reflectivity, $\mathrm{R}_{2}=30 \%$; pump beam radius, $r=1 \mathrm{~mm}$ and pulse duration of the laser pump, $\Delta \mathrm{t}=5 \mathrm{~ns}$. Lasing is achieved when the pump energy is around $3 \mathrm{~mJ}$. The simulation results clearly show that single-pulse generation was observed at the pump energy near to threshold and the output laser pulse duration becomes shorter as the pump energy increases. The shortest pulse duration that can be achieved without damaging the crystal is about $349 \mathrm{ps}$ at $8 \mathrm{~mJ}$ pump energy (Fig. 3). However, multi-pulse generation of relaxation oscillations was observed when pump energy is larger than $8 \mathrm{~mJ}$. 
The Ce: LiCAF laser could generate shorter laser pulse when its pump pulse duration at $266 \mathrm{~nm}$ is shorter. For example, a Ce:LiCAF laser that uses a 10-mm long Ce:LiCAF crystal, a $15-\mathrm{mm}$ long cavity and an output coupler of $30 \%$ reflectivity, generated single UV pulses of 150 ps when the pump pulse at $266 \mathrm{~nm}$ of 75 ps duration [19].

Generation of single 31.5 ps laser pulses of $10 \mu \mathrm{J}$ pulse energy and spectral bandwidth of $2.8 \mathrm{~nm}$ at $290 \mathrm{~nm}$ was achieved with the cavity transient method (Fig. 4). The low-Q, 2-cm cavity laser oscillator was constituted with a $1 \mathrm{~mm}, 1 \mathrm{~mol} \% \mathrm{Ce}^{3+}$-doped LiCAF crystal and a output coupler of $30 \%$ reflectivity. The laser was pumped by 75 ps pulses of $140 \mu \mathrm{J}$ pump energy and has feasible slope efficiency of about $10 \%$ [16].
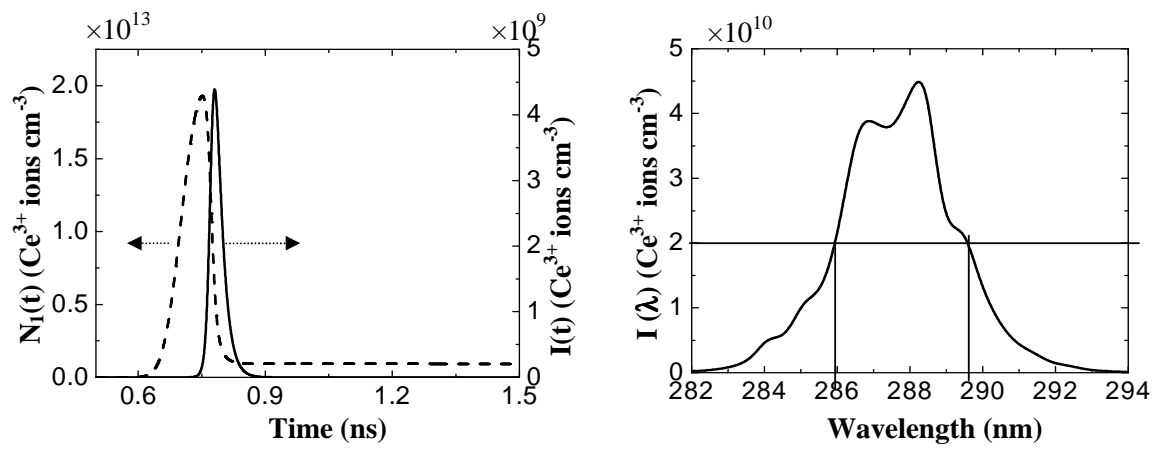

Fig. 4. Generation of single short pulse and the spectro-profile of the broadband, shortpulse Ce:LiCAF laser emission from the low-Q $\left(\mathrm{R}_{2}=30 \%\right)$, short cavity $(\mathrm{L}=2 \mathrm{~mm})$ oscillator. The 1 -mm-long, $1 \mathrm{~mol} \% \mathrm{Ce}^{3+}$-doped LiCAF crystal pumped with a $266 \mathrm{~nm}$, 75 ps pulse [16].

For some solid-state gain media like Ce:LiCAF, short laser pulse generation basing on mode-locking methods has been rather slowly developed due to the difficulty of obtaining continuous wave $(\mathrm{CW})$ laser operation, which is required for Kerr lens mode-locking schemes utilizing spatial or temporal Kerr type nonlinearity. Furthermore, the short laser upper level lifetime of Ce:LiCAF (about $25 \mathrm{~ns}$ ) means that high pump power densities are required to achieve $\mathrm{CW}$ and mode-locked laser operation. Recently, Eduardo Granados et. al, successfully developed a 4mirror mode-locked Ce:LiCAF laser to generate UV pulses of 6 ps using of synchronous pump method. The Ce:LiCAF laser has a maximum output power of $52 \mathrm{~mW}$ and a $13 \%$ slope efficiency in the deep UV with output emission centered at $291 \mathrm{~nm}$ [22].

To produce high pulse energy, larger Ce:LiCAF laser crystal would be required. For example, a large Ce:LiCAF crystal of $15 \mathrm{~mm}$ diameter was grown successfully by the Czochralski method and used as an active medium. The Ce:LiCAF laser generated short pulses of energy as high as $21 \mathrm{~mJ}$ at $290 \mathrm{~nm}$. Its slope efficiency was $39 \%$ with an absorbed pump energy of $54 \mathrm{~mJ}$ from the fourth harmonic generation of a Q-switched Nd:YAG laser operating at a 10-Hz repetition rate $[20,21]$. 


\section{HIGH-POWER ULTRAVIOLET Ce:LiCAF LASER AMPLIFIER}

With broad gain spectrum and high saturation fluence, Ce:LiCAF crystal is quite attractive for generation and amplifications of short UV laser pulse. In 1997, author's group demonstrated the direct generation and efficient amplification of UV short pulses using master oscillator - power amplifier system [15, 23]. The Ce:LiCAF master oscillator of 15-mm length was formed by a flat high ref lection mirror and a flat output coupler of 70\%-transmission. A 10-mm Brewster-cut, 1\%-doped Ce:LiCAF crystal was used as an active medium. The Ce:LiCAF master laser oscillator was pumped by $15-\mathrm{mJ}, 10 \mathrm{~ns}$ pulse at $266 \mathrm{~nm}$ from a Q-switched Nd:YAG laser. Single 1-ns laser pulses at $289 \mathrm{~nm}$ of energy of 1-mJ were successfully generated.

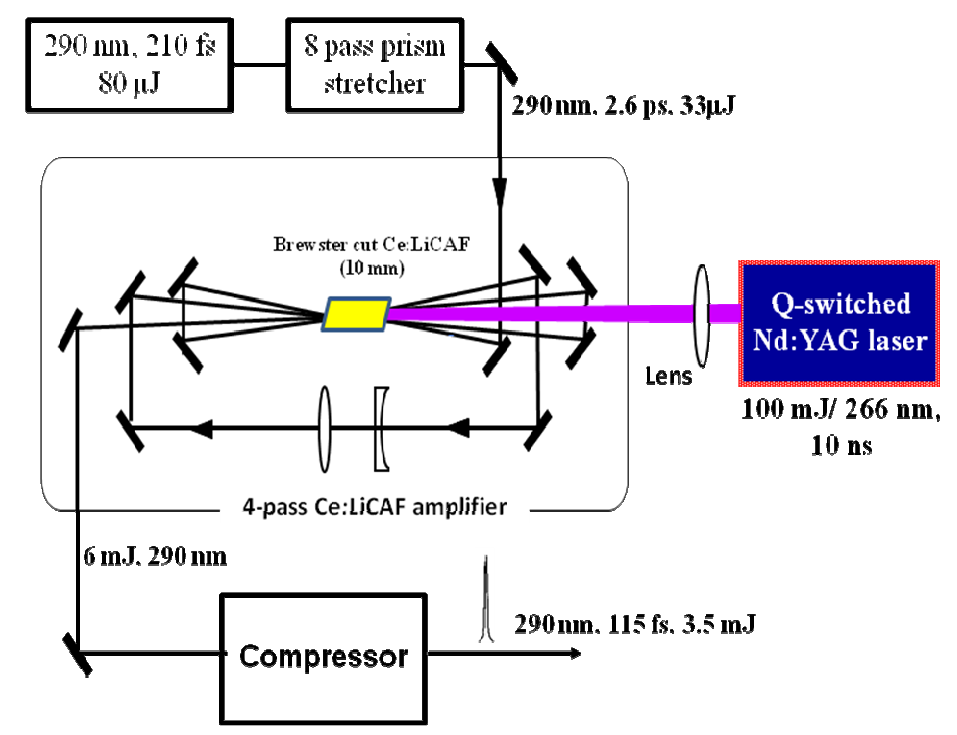

Fig. 5. Experimental setup of the Ce:LiCAF CPA laser system (adapted from $[24,25]$ )

For the power-amplifier, various multipass Ce:LiCAF amplifiers have also been developed by author's group [15,23-26]. For example, a 10-mm, 1\%-doped Brewster-cut Ce:LiCAF crystal was employed as an active medium and pumped by $10-\mathrm{ns}$ pulse of $80 \mathrm{~mJ}$ energy at $266 \mathrm{~nm}$ from the same Q-switched Nd:YAG laser. The differential small-signal gain of the double-pass poweramplifier was measured as high as 100 times. The duration of amplified laser pulse observed by a streak camera was $1 \mathrm{~ns}$. The $1-\mathrm{mJ}$ input pulse was amplified to $14 \mathrm{~mJ}$ corresponding to a $14 \mathrm{MW}$ peak power at $289 \mathrm{~nm}[15,23]$.

In 2001, the amplification of femtosecond UV chirped pulses of Ce:LiCAF (high-saturation fluence $115 \mathrm{~mJ} / \mathrm{cm}^{2}$ ) was experimentally demonstrated in a bow-tie style amplifier as shown in Fig. 5 [24, 25]. The seed pulse of $210 \mathrm{fs}, 80 \mu \mathrm{J}$ and a spectrum bandwidth of $1.0 \mathrm{~nm}$ at $290 \mathrm{~nm}$ was stretched to $2.6 \mathrm{ps}, 33 \mu \mathrm{J}$ pulse and then directed to a 4-pass amplifier. The amplifier used a $10-\mathrm{mm}, 1 \mathrm{~mol} \%$ Brewster-cut $\mathrm{Ce}^{3+}$ : LiCAF crystal as an active medium that was pumped by $10 \mathrm{~ns} 100 \mathrm{~mJ}$ laser pulses at $266 \mathrm{~nm}$. Such an amplifier has a small-signal gain as high as 370 . The energy of the amplified pulse could increase up to $6 \mathrm{~mJ}$. Finally, the amplified pulse was 
compressed with a grating pair compressor down to $120 \mathrm{fs}$ pulse of $2.5 \mathrm{~mJ}$ energy, corresponding to a peak power of larger than $20 \mathrm{GW}$, or with a four-prism compressor down to $115 \mathrm{fs}$ pulse of $3.5 \mathrm{~mJ}$ energy and a peak power larger than $30 \mathrm{GW}$.

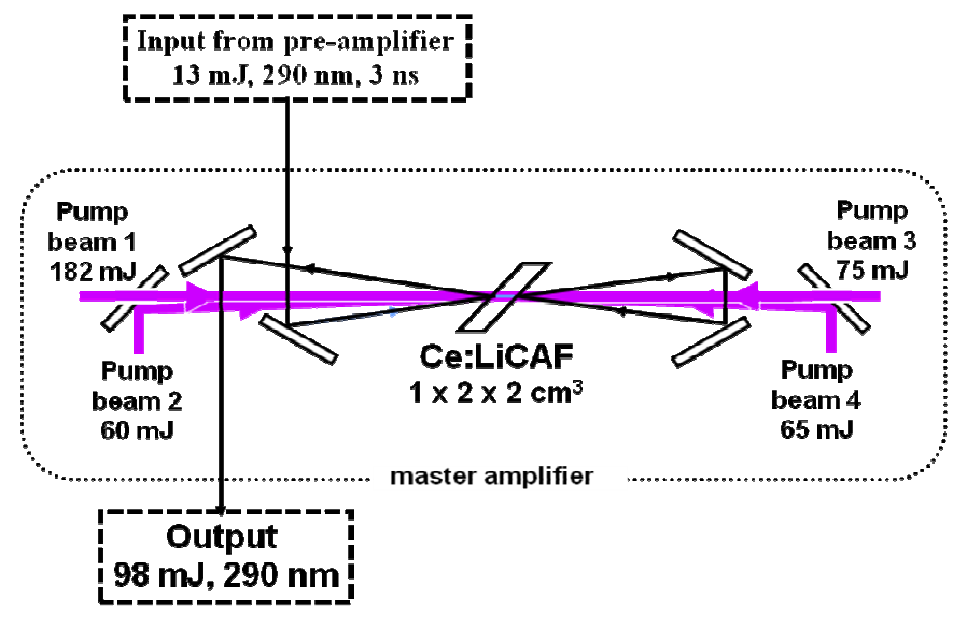

Fig. 6. Double-pass amplifier arrangement for high-energy pulse amplification using larger Ce:LiCAF crystal (adapted from [26]).

However, the size of Ce:LiCAF crystals using in these experiment configuration is still small. To improve amplification, one of the ways is to use larger crystal. A high-energy, allsolid-state, ultraviolet laser power-amplifier module was designed and developed using large-size Ce:LiCAF crystals $\left(1 \times 2 \times 2 \mathrm{~cm}^{3}\right)$. As shown in Fig. 6, a double-pass power amplifier was also developed by Ono et al. to generate up to $98 \mathrm{~mJ}, 3-\mathrm{ns}$ pulses at $290 \mathrm{~nm}$ from $13 \mathrm{~mJ}$ of seed pulse [26]. In the case, the total pump energy is $382 \mathrm{~mJ}$ at $266 \mathrm{~nm}$. The diameters of the seed and pump laser beams into the gain medium are $7 \mathrm{~mm}$ and $8 \mathrm{~mm}$, respectively.

\section{PROPOSALS OF PRODUCING TERAWATT ULTRAVIOLET Ce:LiCAF LASER PULSE}

Generation of high power UV Ce:LiCAF laser emission requires the attention paid simultaneously to gain materials (quality and size) and techniques of high-power short laser pulse generation and amplification. We have investigated and proposed possible improvements producing terawatt Ce:LiCAF ultraviolet laser pulses.

About the direct generation of short UV laser pulse at $290 \mathrm{~nm}$, we have interested in the cavity transient method to produce sub-nanosecond or picosecond UV laser pulses with simple Ce:LiCAF laser master oscillators pumped at $266 \mathrm{~nm}$ by a nanosecond or picosecond lasers. Recently we have demonstrated simple generation of single 31.5 ps UV laser pulse of $10 \mu \mathrm{J}$ pulse energy using the cavity transient method. The investigation was presented in detail in Ref. [16]. The low-Q and short-cavity laser oscillator contains a 1-mm, 1 mol\% $\mathrm{Ce}^{3+}$-doped LiCAF crystal pumped by 75 ps laser pulses and $140 \mu \mathrm{J}$ pump energy. The output picosecond laser pulses of a spectral width of $2.8 \mathrm{~nm}$ at $290 \mathrm{~nm}$ can be as seed pulses and well amplified with a power UV 
laser amplifier system. In the case, this laser oscillator is pumped by a nanosecond laser, subnanosecond UV laser pulses are produced and can be used as seed pulses. Of course, ultrashort UV laser pulses provided from frequency conversion of the primary tunable ultrashort lasers are well used as the seed pulses of a power UV laser amplifier system.

About the gain materials, light amplification by stimulated emission radiation in an amplifier system is directly related to the size and saturation fluence of the gain medium. Moreover, saturation fluence is inversely proportional to the absorption coefficient and consequently, to doping concentration. Therefore, large laser crystals are needed for high peak power laser operation. We demonstrated that with the appropriate crystal size, geometry and doping concentration; combined with multi-high-energy-beam pumping, the development of a UV short pulse amplifier operating at terawatt level would be achievable [25]. The growth method of Ce:LiCAF crystal and other solid state materials has been popularly based on the Bridgman-Stokbarger and Czochralski (CZ) methods [7-26]. To improve the production of laser and scintillating materials, new growth schemes have been proposed and even existing crystal growth techniques are modified. For example, the micro-pulling down ( $\mu$-PD) method of crystal growth is one of the techniques to grow large crystals at a shorter time and at a lower cost compared with other melt growth methods like $\mathrm{CZ}$ or Bridgman. Moreover, this method has the capability of controlling the shape of a grown crystal. A laser quality Ce:LiCAF crystal was successfully grown by the $\mu$-PD method [27-29]. Furthermore, we evaluated the capability of this growth method to grow laser quality crystal by measuring laser emission at $290 \mathrm{~nm}$ with slope efficiency of $23 \%$ [27]. We also reported about UV femtosecond pulse amplification using this crystal and proposed a new configuration of a prismatic cell-pumping to achieve uniform distribution of pumping energy inside the crystal (Fig.7). This is the first demonstration of lasing in the UV region from a $\mu$-PD-method-grown fluoride crystal [27-29].

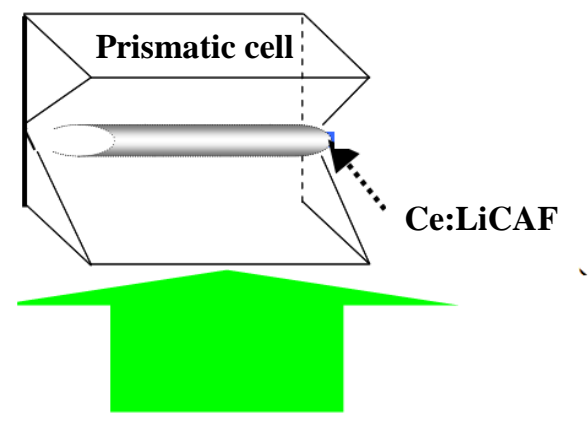

Laser pump

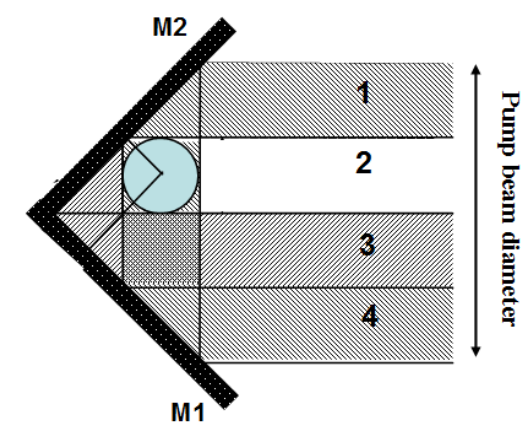

Fig. 7. Experimental setup of creating a uniform distribution of pumping energy inside the ultraviolet Ce:LiCAF laser crystal.

About pumping laser sources of Ce:LiCAF ultraviolet laser and amplifier, high power nanosecond Q-switched Nd:YAG lasers have been used effectively and conveniently under their fourth harmonic generation to provide high power laser pulses at $266 \mathrm{~nm}$. In order to obtain an effective fourth harmonic generation increasing its energy at $266 \mathrm{~nm}$, the authour's group had 
proposed a three $\mathrm{Li}_{2} \mathrm{~B}_{4} \mathrm{O}_{7}$ crystal configuration to generate the fourth harmonics of a high power Q-switched Nd:YAG laser. This provided pulse energy as high as $430 \mathrm{~mJ}$ at $266 \mathrm{~nm}$, corresponding to a total conversion efficiency of $30.5 \%$ [30].

About power UV Ce:LiCAF amplifier, we have been interested in the amplifier configurations presented in Refs. [24, 25] and proposed the experiment of a terawatt all solid state Ce:LiCAF ultraviolet laser - amplifier system as shown in Fig. 8. Such a system contains 03 parts: 1) A laser master oscillator generating single picosecond pulses at $290 \mathrm{~nm}$; 2) A 4-pass large-size Ce:LiCAF pre-amplifier stage; 3) A 2-pass large-size Ce:LiCAF power amplifier stage.

The Ce:LiCAF master oscillator uses a low-Q and short cavity of 2- $\mathrm{mm}$ long which is formed by a flat high-reflection mirror (100\%) and an output coupler of $70 \%$ transmission. A $1 \%$ doped, 1-mm Ce:LiCAF is used as a gain medium and pumped by 75 ps laser pulses and $140 \mu \mathrm{J}$. The laser can produce single $31.5 \mathrm{ps}$ pulses of $10 \mu \mathrm{J}$ energy and a bandwidth of $2.8 \mathrm{~nm}$ [16]. The picosecond laser pulses can be employed as the seed pulses of the pre-amplifier stage.

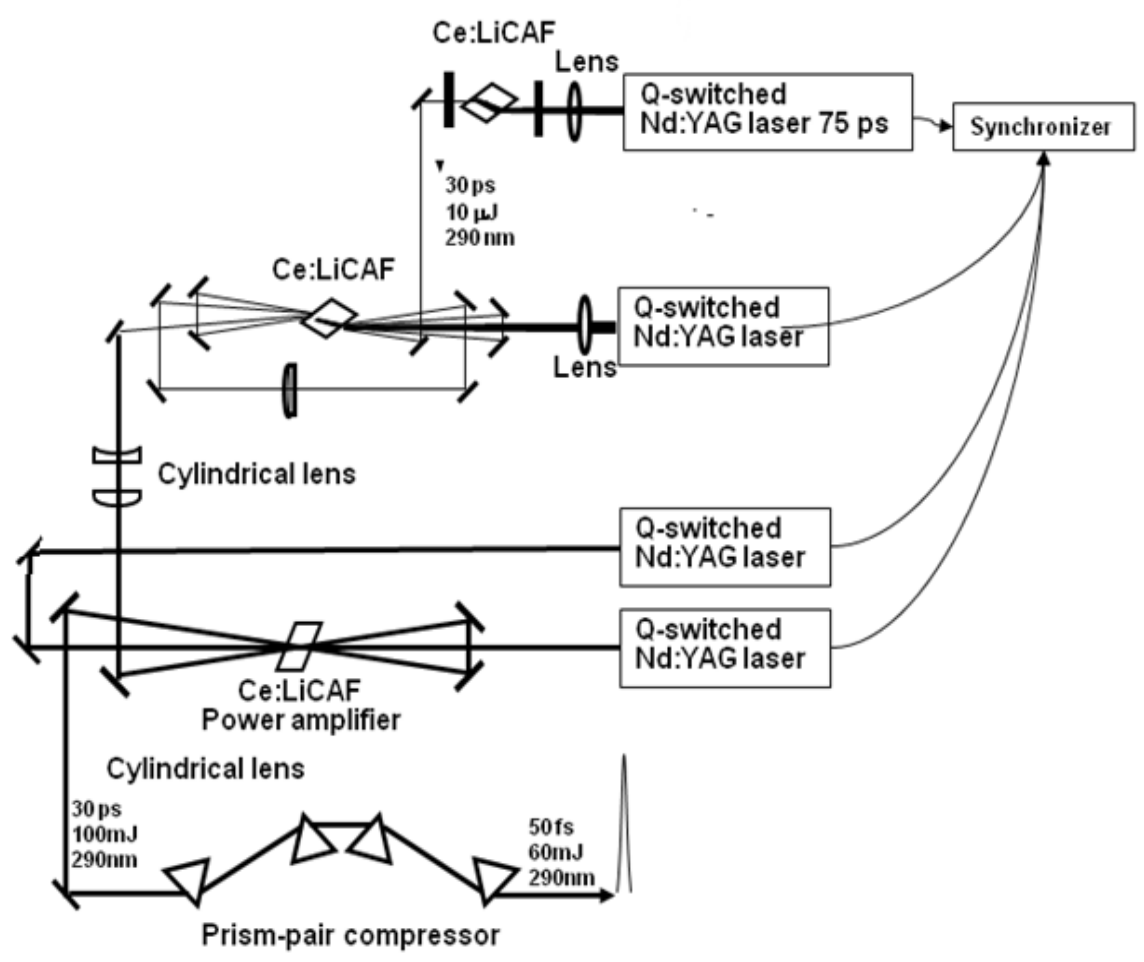

Fig. 8. Experimental setup of terawatt UV Ce:LiCAF laser amplifier: a low-Q, shortcavity master oscillator, pre-amplifier, power amplifier and a four-prisms compressor.

We propose the use of a $1 \times 2 \times 2 \mathrm{~cm}^{3}, 1 \%$ - doped Brewster-cut Ce:LiCAF crystal as gain medium for the pre-amplifier stage. This stage was adapted from the first UV chirped pulse amplification (CPA) [24, 25], however, the size of Ce:LiCAF crystal used for the first UV CPA was rather small $\left(1 \times 1 \times 1 \mathrm{~cm}^{3}\right)$. We use a synchronously operated $10 \mathrm{~Hz}$ Q-switched $\mathrm{Nd}$ : YAG 
laser providing 100-mJ, 266-nm, and 10-ns pulses to pump the pre-amplifier. The pump beam can be softly focused with a 70-cm focal-length lens to a 4-mm diameter to provide a pump fluence of $0.8 \mathrm{~J} / \mathrm{cm}^{2}$, which was well below the damage threshold of Ce:LiCAF crystal $\left(\sim 5 \mathrm{~J} / \mathrm{cm}^{2}\right)$. The diameter of the seed beam is approximately $1.5 \mathrm{~mm}$ for the first and second passes, and expanded it to approximately $3 \mathrm{~mm}$ for the third and fourth passes to avoid deep gain quenching and possible damage to the optics in the amplifier. After the pre-amplifier, the laser pulse energy increases up to several $\mathrm{mJ}$. This laser beam can be expanded to a beam of 7-mm diameter and used as seed pulses for the power amplifier stage which is adapted from Ref. [26].

A large-sized Ce:LiCAF $\left(1 \times 2 \times 2 \mathrm{~cm}^{3}\right)$ is used as gain medium for the double-pass power amplifier stage. To pump the power-amplifier stage, other two Q-switched Nd: YAG lasers are used as pumping with variable Q-switched delay. The total pump energy at $266 \mathrm{~nm}$ for this power amplifier stage is around $0.4 \mathrm{~J}$. In respect to the saturation fluence of Ce:LiCAF and a good spatial overlap between the pump laser and the seed laser beam in the Ce:LiCAF, the diameters of the pump laser and seed beam are $8-\mathrm{mm}$ and $7 \mathrm{~mm}$, respectively.

As a result of strong absorption at $266 \mathrm{~nm}$ of Ce:LiCAF crystal so that almost $100 \%$ of the excitation energy was absorbed in the gain medium, and the pumping density was $0.7 \mathrm{~J} / \mathrm{cm}^{3}$. This pumping condition of less than $0.5 \mathrm{~J} / \mathrm{cm}^{2}$ from each side should provide an adequate safety margin for the optical damage threshold $\sim 2 \mathrm{~J} / \mathrm{cm}^{2}$. The timing of each pump laser was optimized to produce the maximum output. After power amplifier state, the energy of laser pulse reaches to $100 \mathrm{~mJ}$. As the amplified laser pulses of at least $2.8 \mathrm{~nm}$ slightly broadened bandwidth, they would be compressed to $31 \mathrm{fs}$ (assuming a sech ${ }^{2}$ pulse shape with time and bandwidth products of 3.15) with a pulse compressor of double-pass quartz Brewster prism pairs. It is assumed that energy of compressed laser pulse is about $60 \mathrm{~mJ}$, therefore, a peak power of $2 \mathrm{TW}$ is obtainable.

\section{CONCLUSION}

In conclusion, we reviewed the recent progresses in generation and amplification of ultraviolet laser emissions using Ce:LiCAF material as a gain medium. Furthermore, we investigated improvements and proposed possibilities to produce high power short-pulse ultraviolet Ce:LiCAF laser emission. As results, a design of a high power UV picosecond pulse laser - amplifier system was proposed using appropriate Ce:LiCAF crystal size, pumping and resonator geometry and doping concentration as well as combined with multi-high-energy-beam pumping. The UV picosecond pulse laser - amplifier was successfully evaluated toward the production of TW picosecond UV Ce:LiCAF laser emission.

\section{ACKNOWLEDMENT}

The authors would like to thank the support from the Vietnam NAFOSTED under Project (No. 103.06.89.09) and the JSPS-VAST Joint Research Project.

\section{REFERENCES}

[1] P. Misra and M. A. Dubinskii, Ultraviolet Spectroscopy and UV Lasers, Marcel Dekker, Inc (2002); W. Demtroder, Laser Spectroscopy. Basic Concepts and Instrumentation. Third edition. Springer - Verlag, Berlin Heidelberg, 2003.

[2] I. Miyamoto, A. Horn, J. Gottmann, J. Laser Micro/Nanoeng. 2 (2007) 7

[3] S. Pissadakis and M. Konstantaki: Proces. Express 13 (2005) 2605. 
[4] S. McDermit, T. D. Walsh, A. Deslis, and M. L. White, Appl. Opt. 34 (1995) 6201-6210.

[5] D. A. Richter, E. V. Browell, C. F. Butler, and S. H. Noah, "Advanced Airborne UV DIAL System for stratospheric and Tropospheric Ozone and Aerosol Measurements", Advances in Atmospheric Remote Sensing with Lidar, pp. 317-320, Springer, Berlin, 1997.

[6] Y. Mori, I. Kuroda, S. Nakajima, T. Sasaki, and S. Nakai, Appl.Phys. Lett. 67 (1995) 1818.

[7] Mark A. Dubinskii, Vadim V. Semashko, Alexander K. Naumov, Ravil Yu. Abdulsabirov, Stella L Korableva, J. Mod. Opt. 40 (1993) 1-5.

[8] M. A. Dubinskii, V. V. Semashko, A. K. Naumov, R. Y. Abdulsabirov, and S. L. Korableva, Las. Phys. 3 (1993) 216

[9] C. D. Marshall, S. A. Payne, J. A. Speth, W. F. Krupke, G. J. Quarles, V. Castillo and B. H. T. Chai, J. Opt. Soc. Am. B 11 (1994) 2054.

[10] N. Sarukura, M. A. Dubinskii, Z. Liu, V. V. Semashko, A. K. Naumov, S. L. Korableva, R. Y. Abdulsabirov, K. Edamatsu, Y. Suzuki, and T. Segawa, IEEE J. Select. Topics Quantum Electron. 1 (1995) 792.

[11] J. F. Pinto, L. Esterowitz and G. J. Quarles, Electron Lett. 31 (1995) 2009.

[12] T. Kozeki, Y. Suzuki, M. Sakai, H. Ohtake, N. Sarukura, K. Shimamura, T. Fukuda, T. Nakajyo, F. Sakai, and Y. Aoki, Appl. Phys. B. 74 (2002) S185.

[13] Z. Li, N. Sarukura, M. A. Dubinskii, R. Y. Abdulsabirov, S. L. Korableva, A. K. Naumov, and V. V. Semashko, Jpn J. Appl. Phys. 27 (1998) L36-L38.

[14] Nobuhiko Sarukura, Zhenlin Liu, Yusaburo Segawa, Vadim V. Semashko, Alexander K. Naumov, Stella L. Korableva, Ravil Yu. Abdulsabirov, Mark A. Dubinskii, Appl. Phys. Lett. 67 (1995) 602.

[15] Nobuhiko Sarukura, Zhenlin Liu, and Hideyuki Ohtake, Yusaburo Segawa, Mark A. Dubinskii, Vadim V. Semashko, Alexander K. Naumov, Stella L. Korableva, and R. Y. Abdulsabirov, Opt. Lett. 22 (1997) 994.

[16] Minh Hong Pham, Marilou Cadatal-Raduban, Mui Viet Luong, Hai Hoang Le, Kohei Yamanoi, Tomoharu Nakazato, Toshihiko Shimizu, Nobuhiko Sarukura, and Hung Dai Nguyen, Jpn. J. Appl. Phys. 53 (2014) 062701.

[17] David W. Coutts and Andrew J. S. McGonigle, Cerium-Doped Fluoride Lasers, IEEE J. Quantum Electron. 40 (2004) 1430

[18] Zhenlin Liu, and Hideyuki Ohtake, Nobuhiko Sarukura, M. A. Dubinskii, V. V. Semashko, A. K. Naumov, S. L. Korableva, R. Y. Abdulsabirov, Subnanosecond tunable ultraviolet pulse generation from a low-Q, short cavity Ce:LiCAF laser, Jpn. J. Appl. Phys, 36 (1997) pp. L1384

[19] Zhenlin Liu, Nobuhiko Sarukura, Mark A. Dubinskii, R. Y. Abdulsabirov, and S. L. Korableva, J. Noli. Opt. Phys \& Mate. 8 (1999) 41-54.

[20] Zhenlin Liu et al., Jpn. J. Appl. Phys. 37 (1998) L1318-L1319

[21] Zhenlin Liu, Kiyoshi Shimamura, Kenji Nakano, Tsuguo Fukuda, Toshimasa Kozeki, Hideyuki Ohtake and Nobuhiko Sarukura, Jpn. J. Appl. Phys. 39 (2000) L466-L467

[22] Eduardo Granados, David W. Coutts, and David J. Spence, Opt. Lett. 34 (2009) 1660.

[23] Zhenlin Liu, Nobuhiko Sarukura, M. A. Dubinskii, V. V. Semashko, A. K. Naumov, S. L. Korableva and R. Y. Abdulsabirov, Jpn. J. Appl. Phys. 37 (1998) L 36-L 38

[24] Zhenlin Liu, Toshimasa Kozeki, Yuji Suzuki, Nobuhiko Sarukura, Kiyoshi Shimamura, Tsuguo Fukuda, Masahiro Hirano, and Hideo Hosono, Jpn. J. Appl. Phys. 40 (2001) 2308-2309.

[25] Zhenlin Liu, et al., Opt. Lett. 26 (2001) 301.

[26] Shingo Ono et al., Appl. Opt. 41 (2002) 7556.

[27] Pham Hong Minh et al., Jpn. J. Appl. Phys. 47 (2008) 5605.

[28] Pham Hong Minh et al. Communications in Physics 19 Special Issue (2009) 137144.

[29] M. Kouno et al., J. Cryst. Grow. 318 (2011) 737.

[30] Y. Suzuki et al., Jpn. J. Appl. Phys. 41 (2002) L823. 